\title{
Hydrogen cyanide emission in the lung by Staphylococcus aureus
}

\begin{abstract}
To the Editor:
Early detection of Pseudomonas aeruginosa in cystic fibrosis (CF) patients is crucial, since eradication in a later stage is extremely difficult [1]. Until now, it has been assumed that hydrogen cyanide (HCN) can be considered as a specific biomarker for P. aeruginosa in exhaled breath [2-5]. Only Burkholderia cepacia is also known to produce $\mathrm{HCN}$ in vitro $[6,7]$, but $\mathrm{HCN}$ is not a biomarker for B. cepacia complex infection in vivo [8]. To our knowledge, actual proof that $\mathrm{HCN}$ is not produced by other pathogens is lacking. Investigations into the emission of HCN by Staphylococcus aureus, a predominant CF pathogen throughout childhood, have been very limited. In a single report, five strains have been shown to produce low values in vitro [9], and in vivo studies have not been reported.
\end{abstract}

We therefore measured the real-time HCN production by clinical $S$. aureus isolates in vitro, and HCN levels in the exhaled breath of $S$. aureus-infected CF patients, in comparison with non-infected CF patients, as well as healthy persons as controls. Furthermore, we investigated whether the $S$. aureus strains cultured from patients in whom HCN was detected in breath, were capable of producing HCN.

Five clinical isolates of $S$. aureus from sputum of $\mathrm{CF}$ patients were inoculated into 50-mL brain-heart infusion broth in an initial concentration of $5 \times 10^{6} \mathrm{CFU} \cdot \mathrm{mL}^{-1}$. The setup used for analysing bacterial headspace (in triplicate) and determination of growth curves has been previously described.

CF patients (aged $\geqslant 6$ years old) were recruited from the outpatient clinic of Radboud University Medical Center and Erasmus Medical Center (Nijmegen and Rotterdam, the Netherlands, respectively). This study was approved by the local medical ethics committee (CMO - Nijmegen/Arnhem) which waived written informed consent. Oral consent was obtained from participants aged $\geqslant 12$ years and from parents of children aged $6-18$ years.

We included nonsmoking, infected and non-infected CF patients and healthy controls. Infection was defined as culturing $S$. aureus, P. aeruginosa or B. cepacia in sputum on the day of breath sampling or in $>50 \%$ of sputum samples (minimum of four samples) over the previous 12 months. Free of infection was defined as no $S$. aureus, P. aeruginosa or B. cepacia in sputum samples (minimum of four samples) over the previous 12 months. Sputum samples were obtained as a part of routine clinical care. Before sampling, all participants rinsed their mouth with water. Each subject provided two single mouth-exhaled breath samples that were collected in separate 3-L Tedlar bags (ProCare B.V., Groningen, the Netherlands) at a constant flow rate of $300 \mathrm{~mL} \cdot \mathrm{s}^{-1}$ using a breath sampler (Loccioni, Angeli di Rosora, Italy) [10]. Briefly, the participants exhaled through a bacterial filter (Air Safety Limited, Morecambe, UK) and a non-rebreathable T-piece (Vacumed, Ventura, CA, USA) which were connected to a $\mathrm{CO}_{2}$ sensor and a calibrated buffer pipe. From the total exhalation, the dead space air $(150 \mathrm{~mL})$ was discarded and the remaining part collected in the sampling bag. The breath samples were collected $90 \mathrm{~s}$ apart and stored for a maximum of $8 \mathrm{~h}$ prior to analysis. Quantification of $\mathrm{HCN}$ in breath was performed using laser-based photoacoustic spectroscopy as described earlier $[8,11]$. Exhaled HCN levels were normalised to respiratory $\mathrm{CO}_{2}$ concentration and mean values were considered for analysis.

To validate whether the particular $S$. aureus strains from randomly selected $S$. aureus-infected patients in whom $\mathrm{HCN}$ was detected in breath were capable of producing $\mathrm{HCN}$, we measured the HCN production from these five separately cultured $S$. aureus strains. The strains were isolated from sputum that was collected on the day of breath sampling. The total production of HCN in vitro over $30 \mathrm{~h}$ was compared with the level in breath of the corresponding patients.

All S. aureus clinical isolates in vitro produced $\mathrm{HCN}$, although the production level was different across the strains (figure 1a). Production started at approximately $3 \mathrm{~h}$, reached a maximum at $6-7 \mathrm{~h}$, and decreased towards the detection limit $24-30 \mathrm{~h}$ after inoculation. The absolute production ranged from (mean \pm SD) $45.8 \pm 37.4$ (isolate 3) to $994.2 \pm 807.0 \mathrm{nmol}$ (isolate 2 ) over $30 \mathrm{~h}$.

The growth of all S. aureus strains was monitored for $48 \mathrm{~h}$ from the time of inoculation (data not shown). All strains showed comparable concentrations in the stationary phase $\left(10^{8}-10^{9} \mathrm{CFU} \cdot \mathrm{mL}^{-1}\right)$. Clinical isolate 1,2 and 3 reached the stationary phase at $16 \mathrm{~h}$ after inoculation, while number 4 and 5 grew slower and reached the stationary phase after approximately $20 \mathrm{~h}$.

We included 17 CF patients infected with S. aureus (10 with S. aureus in sputum on the day of breath sampling and seven with $S$. aureus in $>50 \%$ of sputum samples, median (interquartile range (IQR)) age 15 (11-31.5) 

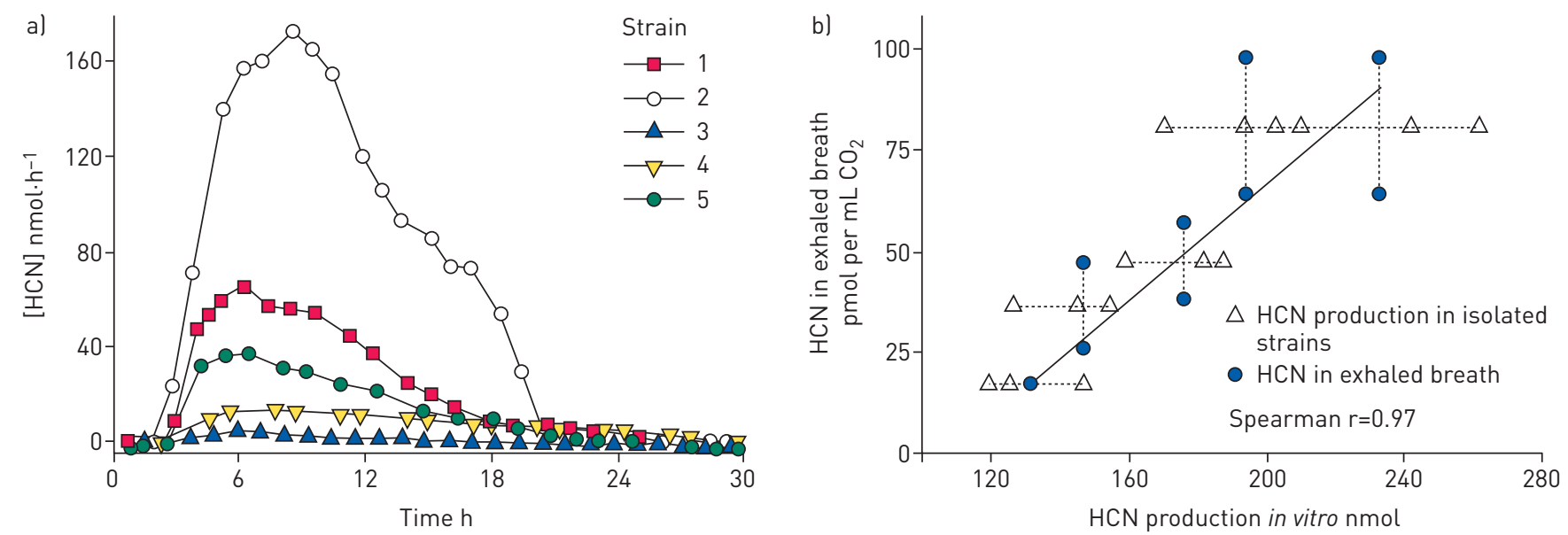

FIGURE 1 Hydrogen cyanide (HCN) production of 10 Staphylococcus aureus strains. a) In vitro production rates of five clinical S. aureus strains from sputum of cystic fibrosis patients. b) HCN production by five $S$. aureus strains isolated from sputum of cystic fibrosis patients (other than those in a) collected on the day of breath sampling in triplicate versus the concentration of $\mathrm{HCN}$ in exhaled breath in duplicate.

years), and a combined group of eight CF patients free from $S$. aureus infection and 12 healthy controls (median (IQR) age 24 (21-28) years). Exhaled HCN levels of S. aureus infected CF patients, were significantly higher compared to the combined group of non-infected CF patients and healthy controls (median (IQR) 38.0 (25.9-71.3) versus 21.9 (6.8-32.8) $\left.\mathrm{pmol} \cdot \mathrm{mL}^{-1} \mathrm{CO}_{2}, \mathrm{p}=0.01\right)$. No statistical difference was found for $\mathrm{HCN}$ levels in breath of non-infected CF patients compared to healthy controls (median (IQR) 21.7 (7.0-48.6) versus 19.6 (3.4-30.7) $\mathrm{pmol} \cdot \mathrm{mL}^{-1} \mathrm{CO}_{2}, \mathrm{p}=0.85$ ).

The HCN production levels of the $S$. aureus strains isolated from sputum on the day of breath sampling were higher compared to breath HCN levels and were strongly correlated (Spearman $\mathrm{r}=0.97$ ) (figure 1b).

This study demonstrates that $\mathrm{HCN}$ is produced by $S$. aureus in vitro and is present in exhaled breath of S. aureus infected CF patients. GiLChRIST et al. [9] cultured five strains of $S$. aureus on blood agar and reported HCN concentrations of 1-6 parts per billion by volume at 24, 48, 72 and $96 \mathrm{~h}$, which are lower compared to those measured in our study. Variations in strain, culturing method (planktonic versus biofilm conditions), medium (brain-heart infusion versus blood agar) or sampling time-points may account for these differences. By comparing the HCN production dynamics with bacterial growth curves it can be concluded that $S$. aureus starts to produce $\mathrm{HCN}$ in the exponential growth phase and varies between strains. $\mathrm{HCN}$ is a highly toxic compound, well-known to inhibit cellular respiration [12]. We speculate that the differences in absolute production may contribute to differences in virulence between $S$. aureus strains, since HCN is associated with lung damage, impaired lung function [13] and reduction of the host response effectiveness in CF [11]. Therefore, patients with elevated HCN levels might benefit from aggressive antibiotic treatment in early CF lung disease. The HCN levels in S. aureus infected patients are significantly higher compared to a combined group of non-infected patients and healthy controls. The correlated HCN levels in vitro and in breath indicate that the breath HCN levels originate from S. aureus infection in the lung.

Our results show that HCN in exhaled breath is not an exclusive biomarker for P. aeruginosa infection, which is a significant drawback in the attempt to develop a specific diagnostic tool for early detection of P. aeruginosa in patients with CF.

@ERSpublications

Hydrogen cyanide is produced by $S$. aureus in vitro and in vivo and is not an exclusive biomarker for P. aeruginosa http://ow.ly/4nsh7y

Anne H. Neerincx ${ }^{1,2}$, Ylona A.M. Linders ${ }^{1,2}$, Laura Vermeulen ${ }^{1}$, Robert A. Belderbos ${ }^{3}$, Julien Mandon ${ }^{1}$, Esther van Mastrigt ${ }^{3}$, Marielle W. Pijnenburg ${ }^{3}$, Jakko van Ingen $^{4}$, Johan W. Mouton ${ }^{4,5}$, Leo A.J. Kluijtmans ${ }^{6}$, Ron A. Wevers ${ }^{6}$, Frans J.M. Harren ${ }^{1}$, Simona M. Cristescu ${ }^{1,8}$ and Peter J.F.M. Merkus ${ }^{2,7,8}$

${ }^{1}$ Dept of Molecular and Laser Physics, Institute for Molecules and Materials, Radboud University, Nijmegen, The Netherlands. ${ }^{2}$ Dept of Paediatrics, Amalia Children's Hospital, Radboud University Medical Center, Nijmegen, The Netherlands. ${ }^{3}$ Dept of Paediatrics, Erasmus University Medical Centre-Sophia Children's Hospital, Rotterdam, The Netherlands. ${ }^{4}$ Dept of Medical Microbiology, Radboud University Medical Center, Nijmegen, The Netherlands. ${ }^{5}$ Dept of Medical Microbiology and Infectious Diseases, Erasmus Medical Center, Rotterdam, The Netherlands. ${ }^{6}$ Dept of Laboratory Medicine, Translational Metabolic Laboratory, Radboud University Medical Center, Nijmegen, The Netherlands. ${ }^{7}$ Dept of Paediatrics, Canisius Wilhelmina Hospital, Nijmegen, The Netherlands. ${ }^{8}$ Both authors contributed equally. 
Correspondence: Simona M. Cristescu, Dept of Molecular and Laser Physics, Institute for Molecules and Materials, Radboud University, Heyendaalseweg 135, 6525 AJ, Nijmegen, The Netherlands. Email: s.cristescu@science.ru.nl

Received: Dec 112015 | Accepted after revision: April 252016 | First published online: May 262016

Support statement: Funded by Longfonds (project No 3.3.11.002). Funding information for this article has been deposited with Open Funder Registry.

Conflict of interest: None declared.

\section{References}

1 Taccetti G, Campana S, Festini F, et al. Early eradication therapy against Pseudomonas aeruginosa in cystic fibrosis patients. Eur Respir J 2005; 26: 458-461.

2 Enderby B, Smith D, Carroll W, et al. Hydrogen cyanide as a biomarker for Pseudomonas aeruginosa in the breath of children with cystic fibrosis. Pediatr Pulmonol 2009; 44: 142-147.

3 Gilchrist FJ, Bright-Thomas RJ, Jones AM, et al. Hydrogen cyanide concentrations in the breath of adult cystic fibrosis patients with and without Pseudomonas aeruginosa infection. J Breath Res 2013; 7: 026010.

4 Smith D, Španěl P, Gilchrist FJ, et al. Hydrogen cyanide, a volatile biomarker of Pseudomonas aeruginosa infection. J Breath Res 2013; 7: 044001.

5 Gilchrist FJ, Belcher J, Jones AM, et al. Exhaled breath hydrogen cyanide as a marker of early Pseudomonas aeruginosa infection in children with cystic fibrosis. ERJ Open Res 2015; 1: 00044-2015.

6 Neerincx AH, Mandon J, van Ingen J, et al. Real-time monitoring of hydrogen cyanide (HCN) and ammonia (NH3) emitted by Pseudomonas aeruginosa. J Breath Res 2015; 9: 027102.

7 Ryall B, Lee X, Zlosnik JE, et al. Bacteria of the Burkholderia cepacia complex are cyanogenic under biofilm and colonial growth conditions. BMC Microbiol 2008; 8: 108.

8 Gilchrist FJ, Sims H, Alcock A, et al. Is hydrogen cyanide a marker of Burkholderia cepacia complex? J Clin Microbiol 2013; 51: 3849-3851.

9 Gilchrist FJ, Alcock A, Belcher J, et al. Variation in hydrogen cyanide production between different strains of Pseudomonas aeruginosa. Eur Respir J 2011; 38: 409-414.

10 Thornadtsson A, Neerincx A, Hogman M, et al. Extended NO analysis may improve personalized anti-inflammatory treatment in asthmatic children with intermediate FENO50. J Breath Res 2015; 9: 047114.

11 Anderson R, Roddam L, Bettiol S, et al. Biosignificance of bacterial cyanogenesis in the CF lung. J Cyst Fibros 2010; 9: 158-164.

12 Williams HD, Zlosnik JEA, Ryall B. Oxygen, cyanide and energy generation in the cystic fibrosis pathogen Pseudomonas aeruginosa. Adv Microb Physiol 2007; 52: 1-71.

13 Nair C, Shoemark A, Chan M, et al. Cyanide levels found in infected cystic fibrosis sputum inhibit airway ciliary function. Eur Respir J 2014; 44: 1253-1261.

\section{Pouched rats as detectors of tuberculosis: comparison to concentrated smear microscopy}

To the Editor:

In 2014, 1.5 million people died of tuberculosis (TB), a disease that can be cured in nearly every case if detected in time. Rapid and accurate detection of TB is a crucial component of the World Health Organization's 2016-2035 End TB Strategy [1]. Pouched rats, Cricetomys ansorgei (previously called Cricetomys gambianus [2]), are able to detect Mycobacterium tuberculosis by sniffing sputum samples [3]. Since 2007, they have been used for second-line screening of sputum samples previously evaluated by ZiehlNeelsen microscopy $(\mathrm{ZN})$ at clinics in Tanzania. Use of the rats increases new case detections by $\sim 40 \%$ [3].

In 2013, a TB-screening programme using rats opened in Maputo (Mozambique). Here, as in Tanzania, presumptive TB patients provide two samples, which the rats evaluate by sniffing holes above pots containing the heat-inactivated sputum. The rats are taught to pause above samples deemed TB positive by ZN. Samples deemed TB negative by ZN but TB positive by rats are evaluated by concentrated smear light-emitting diode fluorescence microscopy (LED-FM) $[4,5]$. Presumptive TB patients with at least one LED-FM-positive sputum sample are assumed to have active TB and constitute additional case detections. Information about the disease status of such patients is conveyed to clinics so that they can be treated. Although sputum concentration and the use of LED-FM (rather than $\mathrm{ZN}$ ) result in higher sensitivity, TB clinics in Mozambique and other financially impoverished countries are rarely able to implement these methods due to resource constraints $[5,6]$. 